for very feeble magnetizing forces, iron may be diamagnetic That suggestion was confessedly speculative; its basis was the notion that the Weber-Ampere electro-magnetic molecules suffer something akin to static friction when the process of magnetization attempts to bring them into alignment. Since it was thrown out, Lord Rayleigh has proved that the susceptibility of iron is constant, and has a fairly high positive value, for magnetic forces ranging from 0.03 to 0.04 C.G.S. downwards. Below the lowest force he has investigated, it is still conceivable that there may be a change in the susceptibility, but it is extremely improbable. In all likelihood, Lord Rayleigh's straight line in the curve of $\mathrm{B}$ and $\mathrm{H}$ or of $\mathrm{I}$ and $\mathrm{H}$ extends back to the origin. This at least is certain, that if there is any region at the beginning of magnetization within which the permeability is less than unity, or even no more than unity, it must be so infinitesimally narrow that its existence has no practical interest. For such magnetic forces as act on a lightning-conductor when a discharge is passing, iron is, beyond any question, strongly paramagnetic, and the self-induction with the iron conductor consequently greater than with the copper.

J. A. Ewing.

Dundee, May II.

\section{Notes on the Reproduction of Rudimentary Toes in Greyhounds.}

AT the present writing, I bave under my observation a fine male, light clay-coloured, smooth haired greyhound, which at certain intervals well illustrates the reproduction of the rudimentary digits of its feet, after they have been accidentally amputated. To-day this dog has growing on the inner aspects of both its fore and hind feet, and situated some 9 centimetres above the soles, on each limb, a strong rudimentary toe. If we choose, say, this toe on the right hind foot as an example of them all, we find it to be loosely attached, rather more than a centimetre long to the base of the claw, which latter is large and strong, powerfully curved, and fully as big as any of the claws on the foot phalanges. I further find that this toe has a well-marked pad on its under side, but careful examination fails to detect any bone in the proximal joint, from which I also infer that the ungual phalanx likewise lacks one, though this is not so easily determined without cutting through the horny theca forming the claw. About four months ago this dog was coursing hares over the prairie of this region, which chances to be overgrown with a stiff growth of sage-brush, about 2 feet to 3 feet high. The wiry stems of this plant, as the dog bounded among them, snipped of all four of these rudimentary digits, close down to the leg in each case, as nicely as though it had been done with a knife, leaving linear wounds about half a centimetre long. Now, instead of the lips of these wounds healing across, as one would naturally suppose they would, they immediately form the basis, in each case, for the growth of another rudimentary clawed toe, fully as perfect as the one which originally sprang from the same site. These subsequent growths take about three months to attain their full size again, when they are very likely to be removed by a similar process, and once more grow out as before, and so on indefinitely.

From several points of view, this case, as occurring in a vertebrate so high in the scale as a dog, has interested me very much indeed, and $I$ further find that it is no uncommon thing to meet with greyhounds that have never possessed these rudimentary pollices and halluces, and it is fair to presume that in this race they are gradually disappearing.

Fort Wingate, New Mexico, March 28.

$$
\text { R. W. SHuFEI,DT. }
$$

\section{Dreams.}

In discussing the differences between dreams and real life, Schopenhauer expresses the opinion that the distinction between these two activities of our representative power consists merely in the possibility of the representations of real life being connected in an uninterrupted successive series, while dreams resemble the separate pages of a book torn asunder, and put together again in complete confusion. Some personal observations of my own do not quite agree with this view. I have watched my dreains for some years, and have remarked that many of them are connected with one another in separate series. It happens to me very often that my dreams consist of a series of representations logically developed (although sometimes the logic is absurd) from other series of representations dreamed long before. It would be interesting to know if anyone else has observed anything of this kind.

Oostkamenogorsk, Western Siberia, April 6.

\section{"Antagonism."}

Mr. Collins (Nature, May 3, p. 7) claims that $\mathrm{Mr}$. Herbert Spencer anticipated Sir Wm. Grove and Prof. Huxley in the expression of the idea of antagonism. I think that priority to all of them must be given to the author of Ecclesiasticus in the Apocrypha, who says (chap. xlii., verse 24), "All things are double, one against the other. He hath made nothing imperfect."

Parsonstown, May I3.

SUGGESTIONS ON THE CLASSIFICATION OF THE VARIOUS SPECIES OF HEAVENLY BODIES. ${ }^{1}$

V.

\section{Classification into Species.}

WE are now in a position to apply all that has gone before in a summarized statement of the various spectral changes, including those connected with hydrogen, which take place not only in these objects studied by Dunér, but in those others to which I have referred as forming the true beginning of the group.

The following sţatement, however, must not be taken as anything else than a first approximation to the real criteria of specific differences. I am convinced that further thought is required on it, and that such further thought will be well repaid.

\section{The Sequence of the Various Bands in the Spectra of the Elements indicated by Bodies of the Group.}

In comparing the spectrum of an element which has been mapped in the laboratory with the absorption bands in the spectrum of a "star," we need only consider those bands and flutings which stand out prominently and are the first to flash out when there is only a small quantity present. Thus, in the flame spectrum of barium there is an almost continuous background of flutings with a few brighter bands in the green, and it is only important to consider the bands, as the flutings would mainly produce a general dimming of the continuous spectrum. In order to show at a glance what portions of the spectrum of an element it is most important for us to consider in this discussion, I have reconstructed the map of lowtemperature spectra which I gave in my previous paper, with reference to those elements. which are indicated in the spectra of bodies of Group II. Five orders of intensities are represented, the longest lines, flutings, or bands being the brightest. The lines, flutings, or bands in the lowest horizon, in the case of each element, are those which are seen at the lowest temperature, and which are the first to appear when only a small quantity of substance is present. Those in the upper horizons are the faintest, and are only seen when the temperature is increased, or a considerable amount of the substance is volatilized. The map shows that if there are any indications of magnesium, for instance, in bodies of low temperatures, the fluting at 500 will be seen, possibly without the other flutings or lines. The first indications of manganese will be the fluting at 558 , and so on. Again, on account of the masking effect of the spectrum of one element upon that of another, we may sometimes have an element indicated in a star spectrum, not by the brightest band or fluting in its spectrum, but by the second or even third in brightness; this, of course, only occurs when the darkest band falls on one of the brightest flutings of

1 The Bakerian Lecture, delivered at the Royal Society on April 12, by J. Norman Lockyer, F.R.S. Continued from p. 35 . 


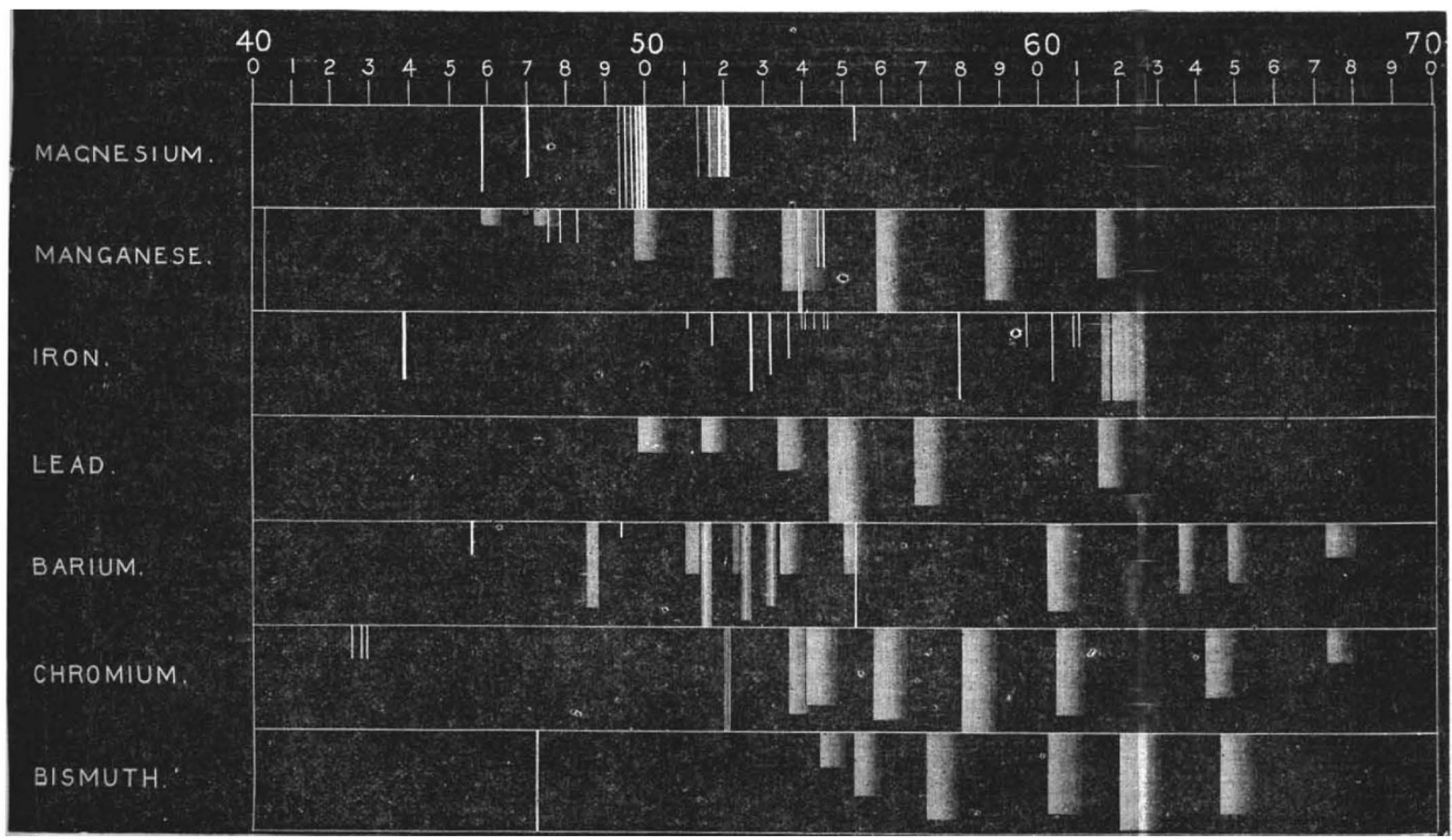

FIG. 8. - Map showing the lines, bands, and flutings seen in the spectra of the elements which are indicated in bodies of Group II. The map is intended to show also the relative intensities of the different lines, bands, and flutings, the lines, \&c., seen in the lowest horizon being those seen at the lowest temperature.

HOT CARBON.

MAGNESIUM.

MANGANESE.

RESULT, SPECIES ?.

HOT CARBON.

MACNESIUM

MANGANESE:

RESULT, SPECIES 3 .

HOT CARBON.

MAGNESIUM.

MANGANESE.

RESULT,

SPECIES 5.
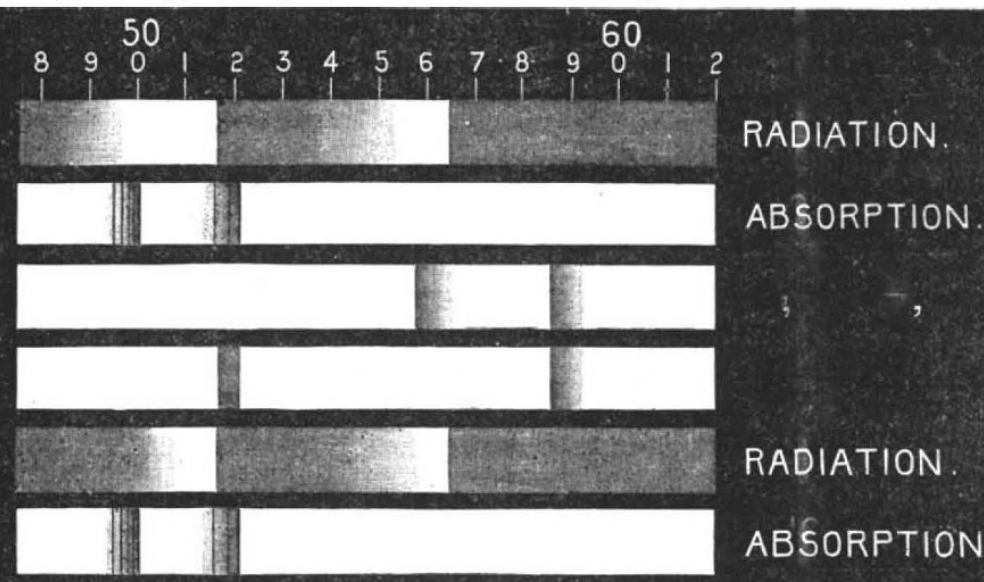

\section{RADIATION}

ABSORPTION.
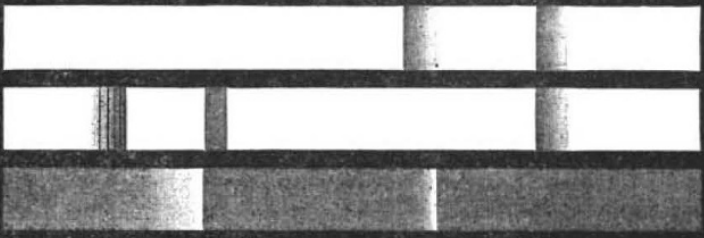

RADIATION.

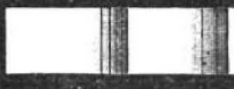

ABSORPTION.

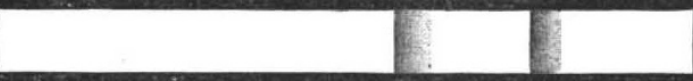

(6)

FIG. 9. - Diagram showing the effects of varations in width of the flutings of carbon upon the integrated spectra of carbon radiation, and magnesium and manganese absorption, as they occur in different species of bodies of Group II. The carbon radiation alone would give bright hands, while the absorption alone would give da: $k$ ones; but if the bright and dark bands fall in the same regions of the spectram, the result will be enfeebled radiation, enfeebled absorption, or nil, according to the relative quantities of radiating and absorbing substances present. Thus, in species 2, the magnesium fluting at 500 is masked by the carbon fluting at 517 , but as the quantity of carbon diminishes, it appears as an absorption band. 
carbon, or upon a dark band in the sfectrum of some other element. In the former case the dark band will be cancelled or masked; in the latter case the two absorptions will be added together, and form a darker band of a different shape.

\section{The Question of Masking.}

If we consider the masking effects of the bright carbon flutings upon the absorption spectrum of each of the elements which, according to the results obtained, enter into the formation of Dunér's bands, we have the following as the main results:--

Magnesium.-There are two flutings of magnesium to be considered, the brightest at 500 and the other at $520 \mathrm{I}$. In the earlier stages of Duner's stars only the fainter one at 5201 is visible, but the absence of the brightest at 500 is accounted for by the masking effect of the bright carbon fluting starting at 517 . As the carbon fades, the 517 fluting narrows and the absorption of magnesium 500 becomes evident.

Manganese.-The two chief flutings of manganese are at 558 and 586 , the former being the brightest fluting in the spectrum. The second fluting is seen in all of Duner's stars. The first fluting, 558 , however, does not appear as an absorption fluting until the radiation fluting of carbon starting at 564 has narrowed sufficiently to unmask it. It is thus easy to understand why, in some stars, there should be the second fluting of manganese without the first.

Barium.-The spectrum of barium consists of a set of flutings extending the whole length of the spectrum, and standing out on this as a background are three bright bands; the brightest band is at 515 , the second is at 525 , and the third, a broader band, is about 485 . The second band is recorded as an absorption band in Dunér's stars, the apparent absence of the first band being due to the masking effect of the bright carbon at 517. The third band at 485 probably forms a portion of band 9. A fourth band, at 533, and the three brightest flutings at 602,635 , and 648 are also seen in $a$ Orionis.

Lead.- The brightest fluting of lead is at 546 . This first appears in species 5 , as a result of increased temperature, and not on account of the removal of any previous mask. The second fluting of lead, at 568 , also appears in two cases.

Chromium.-The flutings of chromium do not form portions of the ten principal bands of Dunerr, but the brightest are seen in $a$ Orionis. The brightest fluting is at 580 , and this forms band I. ; the second, at 557 , is masked by the manganese fluting at 558 , and the third at 536 is seen as line 2. The chromium triplet about 520 , which is visible in the bunsen, is seen as line 3 .

Bismuth.-The brightest fluting of bismuth is at 620 , the second is at $57 \mathrm{I}$, the third at 602 , and the fourth is at 646 . The first is masked by the iron fluting at 615 , the second is probably seen in $a$ Orionis as band II. (570-577).

The points I consider as most firmly established are the masking effects of the bright carbon flutings and the possibility of the demonstration of the existence of some of the flutings in the spectrum by this means, if there were no other. There are two chief cases, the masking of the "nebula" fluting 500 by the bright carbon fluting with its brightest, less refrangible edge at 517 , and that of the strongest fluting of $\mathrm{Mn}=\mathrm{Mn}$ (r) 558 , by the other with its brightest edge at 564. I have little doubt that in some quarters my anxiety not to be content to refer to the second fluting of $\mathrm{Mn}$ without being able to explain the absence of the first one, will be considered thrown away, as it is so easy to ascribe any non-understood and therefore "abnormal" spectrum to unknown physical laws; but when a special research had shown me that at all temperatures at which the flutings of manganese are seen at all, the one at 558 retained its supremacy, I felt myself quite justifred in ascribing its absence in species $1-4$ to the cause I have assigned, the more especially as the $\mathrm{Mg}$ fluting which is visible even in the nebula followed suit.

\section{The Characteristics of the Various Species.}

$I$ append the following remarks and references to the number of the bodies in Dunér's catalogue, in which the specific differences come out most strongly, to the tabular statement. I also refer to some difficulties.

Sp. I. The characteristic here is the almost cometary condition. All three bright carbon flutings generally seen in comets are visible; 474 standing aut beyond the end of the dull blue continuous spectrum of the meteorites, 516 masking $\mathrm{Mg} 500$, and 564 masking $\mathrm{Mn}(\mathrm{I})$ 558. The bands visible in the spectra of bodies belonging to this species will therefore be $\operatorname{Mn}(2) 586$, and $\operatorname{Mg}(2) 521$; band 9 will be so wide and pale that it would most likely escape detection. It is very doubtful whether any of the bodies the spectra of which have hitherto been recorded can be classed in this species, but laboratory work assuredly points to their existence ; it will therefore be extremely interesting if future observations result in their discovery. It is possible, however, that No. I 50 of Dunér's list belongs to this species, but the details are insufficient to say with certainty. His description is as follows:-" 150 . Il me parait y avoir une bande étroite dans le rouge, et une plus large dans le vert" (p. 55).

Sp. 2. Characteristics: appearance of Fe. The number of bands now visible is three-namely, 2,3 , and 7 . The iron comes out as a result of the increased temperature. $M g(I)$ and $M n(I)$ are still masked by the bright carbon flutings, and there is still insufficient luminosity to make the apparent absorption band 9 dark enough to be noticed.

Sp. 3. Characteristics: appearance of $\mathrm{Mg} 500$, which has previously been masked by the carbon bright fluting 517. 8 and 7 are now the darkest bands in the spectrum, 37 .

Sp. 4. Characteristics: appearance of $\mathrm{Pb}(\mathrm{t}) 546$, i.e. band 5. This, if present in the earlier species at all, would be masked by the bright carbon at 564 .

Sp. 5. Characteristics: $\operatorname{Mn}(1)$ is now unmasked. The bands now visible are $2,3,4,5,7$, and 8 , the two latter still 'eing the widest and darkest, because they are essentially low-temperature phenomena.

Sp. 6. Characteristic: band 6, i.e. $\mathrm{Ba}(2), 525$, is now added. The first band of $\mathrm{Ba}$ at 515 is masked by the bright carbon at 517 . The bands now visible are $2-8,7$ and 8 still being widest and darkest. They will all be pretty wide, and they will be dark because the continuous spectrum will be feebly developed.

Sp. 7. Characteristics: appearance of band 9. This, which has been already specially referred to, has been too wide and pale to be observed in the earlier species. Its present appearance is due to the narrowing and brightening of the carbon at 474 and the brightening of the continuous spectrum, the result being a greater contrast. Bands 7 and 8 still retain their supremacy, but all the bands will be moderately wide and dark.

Sp. 8. Characteristics: all the bands $2-9$ are more prominent, so that 7 and 8 have almost lost their supremacy.

Sp. 9. Characteristic: appearance of band I, the origin of which has not yet been determined. All the bands are well seen, and are moderately wide and dark.

Sp. 10. Characteristics : appearance of band 10, and in some cases II. These become visible on account of the brightening of the carbon B fluting and the hydrocarbon fluting at 43I. The spectrum is now at its greatest beauty, and is discontinuous.

Sp. II. Characteristics: the bands are now becoming wider, and 2 and 3 are gaining in supremacy; 7 and 8 become narrower on account of the increased temperature. I and Io are only occasionally seen in this species. 
Sp. I2. Characteristics: with the expansion of the continuous spectrum towards the blue, band 9 becomes very narrow, and cannot be observed with certainty. The other bands, with the exception of 7 and 8 , are becoming wider and paler, while 2 and 3 still gain in supremacy.

Sp. I3. Characteristics: 9 has now entirely disappeared, 2 and 3 still retaining their supremacy.

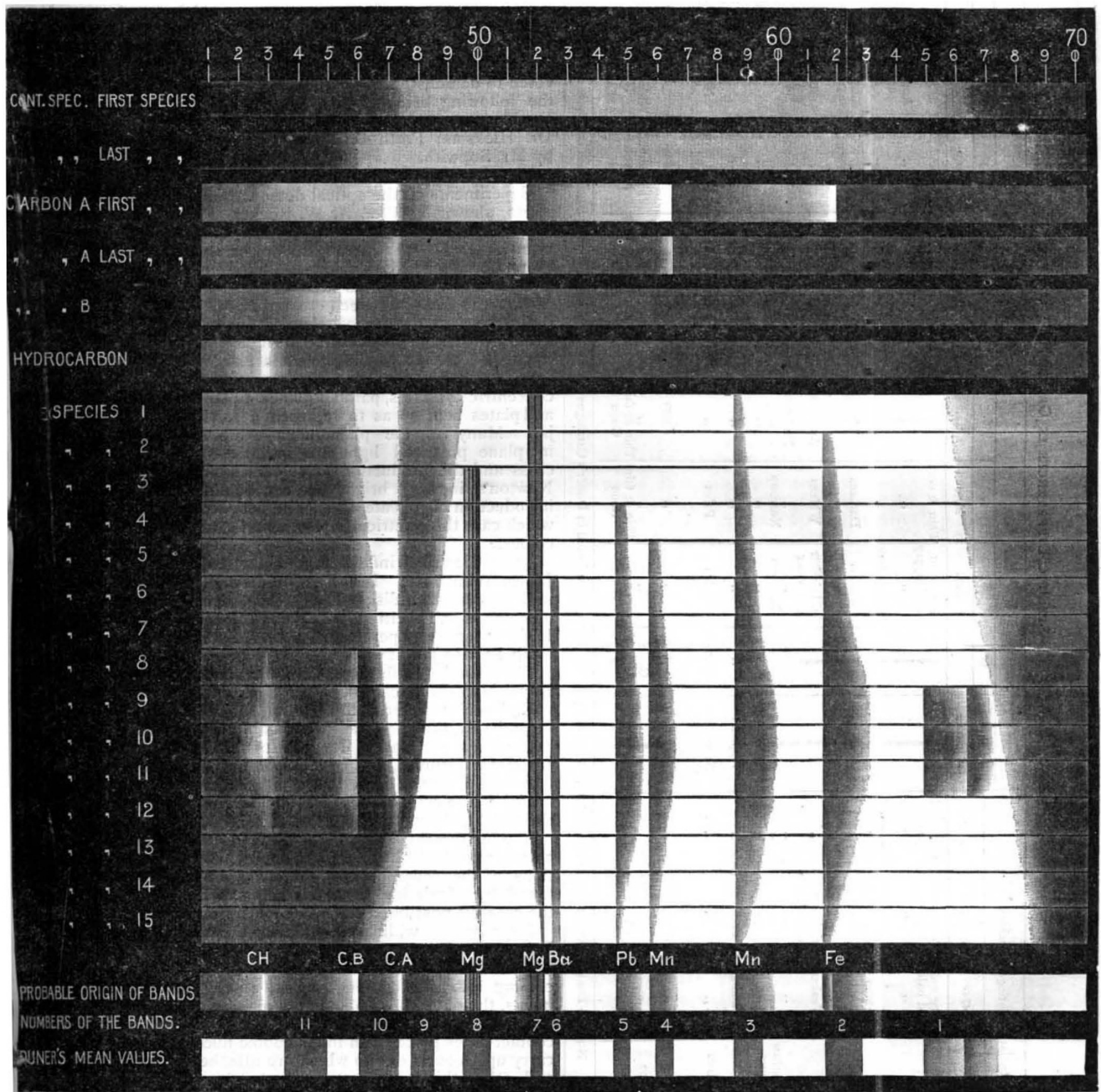

FIG. 10.-Map showing the spectra of the various species of the bolies of Group II., and the probable origin of the bands. The carbon flutings are widest in the first species, and gradually narrow until, in the last species, only a trace of 517 remains. The length of the continuou; spectrum gradually increases as the carbon flutings narrow. The carbon $\mathrm{B}^{1}$ fluting, and the hydrocarbon fluting are only seen in species 8 to 12.

Sp. 14. Characteristics: all the bands are pale and narrow; 2 and 3 will still be darkest, but the difference will not be so great as in the species preceding.

Sp. 15. Characteristics: in ordinary members of this group, 2 and 3 now alone remain visible: they are wide, but feeble, as the continuous spectrum which has been rapidly developing during the last changes is now strong. 


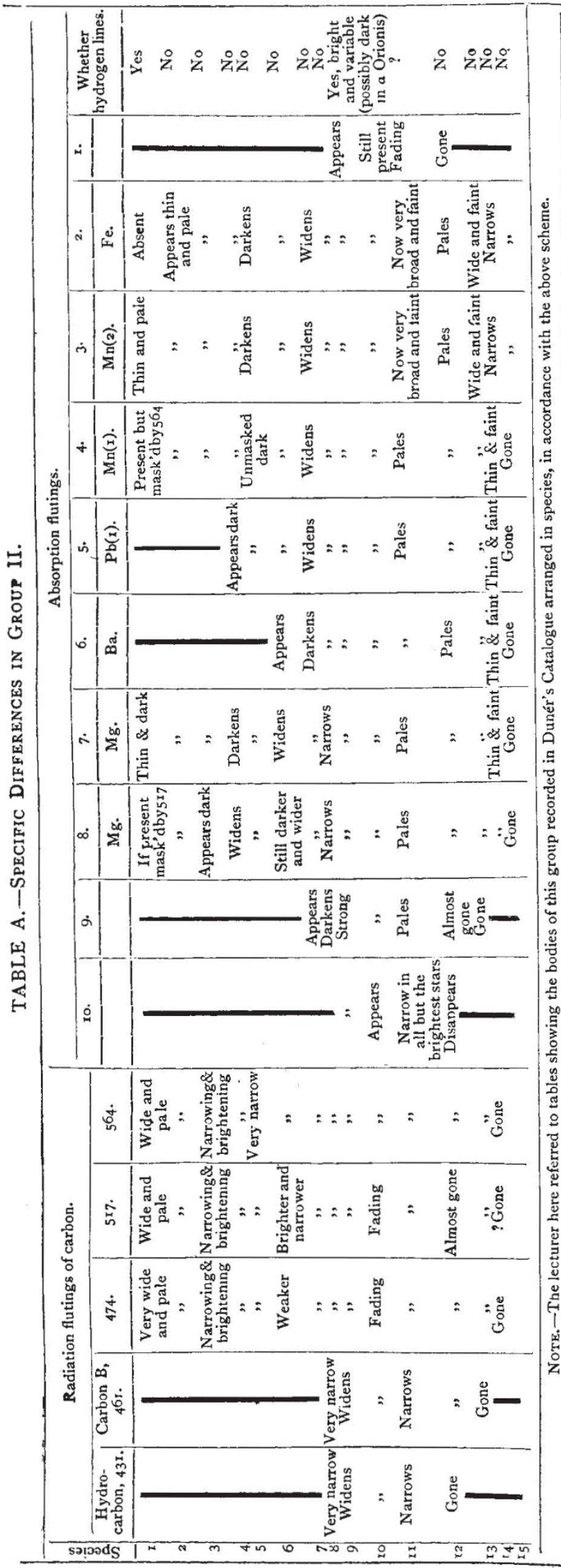

(To te continued.)

\section{THE ROYAL SOCIETY CONVERSAZIONE.}

$T H E$ first conversazione of the season was held on

May 9, and was very numerously attended. More pains than ever seemed to have been bestowed on the arrangements, and the results entirely justified them. As the carefully prepared programme covers eighteen closely printed pages, we can only give a very summary account of the most important demonstrations and exhibits.

Following recent precedents, the meeting-room was devoted to demonstrations by means of the electric lantern, the following being given: image of electric spark, by Dr. Marcet; Mr. Poulton's teeth of Ornithorhynchus, by Dr. Hickson; Forth Bridge, by Mr. Baker; collieries, by Mr. Sopwith.

The chief exhibits in the other rooms were as follow :-

Experiments on the optical demonstration of electrical stress, shown by Prof. A. W. Rücker, F.R.S., and Mr. C. V. Boys. These experiments are similar to those devised by Dr. Kerr, the arrangements being modified so as to render them suitable for exhibition in public. Conductors of various forms are immersed in bisulphide of carbon and placed between crossed Nicol prisms. When the conductors are oppositely electrified the medium is throwninto a state of stress, and the light which had been extinguished by the analyzing prism is restored. The various fo:ms of conductors employed are-parallel cylinders, concentric cylinders, parallel planes, a plane and cylinder, and plates bent so as to represent a section of a Leyden jar. Many of the phenomena exhibited by crystals in plane polarized light are imitated-e.g. the black cross and the production of colours similar to those in Newton's rings. A bright field can be maintained by the introduction of a plate of selenite between the Nicols, in which case the electrical stress is indicated by change of colour.

Large electrical influence machine, exhibited by $\mathrm{Mr}$. James Wimshurst. It has twelve disks of 2 feet 6 inches in diameter ; each disk carries sixteen metal sectors. The machine is self-exciting in any condition of atmosphere. It shows large and perfect brush discharge at its terminals. With Leyden jars it will give sparks $13 \frac{5}{8}$ inches in length,

Photographs of flashes of lightning, exhibited by the Royal Meteorological Society.

Radio-micrometer, exhibited by Mr. C. V. Boys. This is probably the most delicate instrument for measuring radiant heat yet made. It consists of a circuit made of antimony, bismuth, and copper hung by an exceedingly fine fibre of quartz in a strong magnetic field. A scale model of the circuit, twenty times the size or 8000 times the weight, shows the construction of the suspended part of the instrument. The fibre, if magnified to the same extent, would still be finer than spun glass. The proportions of the several parts are those which have been found by calculation (confirmed by experiment) to give the greatest possible delicacy.

Experiments with soap-bubbles, also shown by $\mathrm{Mr}$. Boys. These experiments are arranged to show chiefly the power of an air-film to prevent two bubbles from coming into real contact. Thus, among other experiments, the outer of two bubbles may be pulled out until it squeezes the inner one into a long oval, but no real contact takes place. An inner bubble filled with gas will carry up an outer one to which are attached a wire ring and other things without really touching it at all. A bubble will roll down a spiral groove, also made of soapfilm, or jump one or two steps at a time down a spiral staircase made of soap-film, without touching the spiral film or being injured in the least. Some of the experiments show the effects of diffusion, of vibration, of magnetism, or of electricity upon bubbles or groups of bubbles.

Maps and diagrams illustrative of the recent work of the Geological Survey in the North-West Highlands, exhibited by the Director-General of H.M. Geological 